\title{
Blood Loss in Total Knee Arthroplasty: A Meta-analysis of 4 Different Tourniquet Protocols
}

\author{
Boddu $\mathrm{C}^{1 *}$, Nett MP², Cushner FD $^{3}$ and \\ Scuderi GR ${ }^{4}$ \\ ${ }^{1}$ Clinical Fellow, NSLIJ Adult Reconstruction/Knee, \\ Lenox Hill Hospital, USA \\ ${ }^{2}$ Orthopedic Service Line, Northshore Long Island J ewish \\ Health System, USA \\ ${ }^{3}$ Chief of Orthopedics-Southside Hospital, USA \\ ${ }^{4}$ Program Director, Orthopedic Service Line, USA \\ *Correspondling author: Chandrakanth Boddu, \\ Clinical Fellow, NSLIJ Adult Reconstruction/Knee, Lenox \\ Hill Hospital, USA
}

Received: November 11, 2016; Accepted: February 03, 2017; Published: February 06, 2017

\begin{abstract}
Context: Decreasing perioperative blood loss using a tourniquet during primary total knee arthroplasty (TKA) is a standard practice but there is still no
\end{abstract} consensus on ideal technique.

Objective: We classified and compared the effect of 4 different tourniquet protocols used in primary TKA.

Data Sources: Pub Med and Embase search was performed the term 'Tourniquet Blood loss Knee replacement' to identify original articles.

Study Selection: All the original articles that compared two or more different protocols of tourniquet usage during primary TKA were included. All studies which had an additional variable that would affect perioperative hemoglobin level were excluded.

Data Extraction: Change in the mean hemoglobin level perioperatively when using a specific type of tourniquet was calculated in each study. By combining the results of all studies on each particular type of tourniquet usage, the weighted mean and its variance, standard error, standard deviation and 95\% $\mathrm{Cl}$ were calculated.

Results: 16 studies with a total of 1126 patients were included in metaanalysis. The results of type $2 \mathrm{a}$, type $2 \mathrm{~b}$ and type 3 techniques of tourniquet usage during primary TKA were essentially comparable. Type $2 a$, type $2 b$ and type 3 techniques of tourniquet usage were found to have the lowest mean change in the perioperative hemoglobin level and a non overlapping $95 \%$ confidence interval of the mean change in the perioperative hemoglobin level compared to type 0 technique of tourniquet usage. Type 1 technique of tourniquet usage had the broadest $95 \%$ confidence interval indicating significant individual outliers compared to the rest of the techniques of tourniquet usage during primary TKA.

Conclusion: Type $2 a$, type $2 b$ and type 3 techniques of tourniquet usage are superior to type 0 and type 1 techniques of tourniquet usage in patients undergoing primary TKA.

Keywords: Knee arthroplasty; Blood Loss; Meta-analysis; Tourniquet protocols

\section{Introduction}

Minimizing perioperative blood loss during total knee arthroplasty (TKA) remains a major concern and the specific tourniquet protocol employed during TKA can affect both intraoperative and total perioperative blood loss. The use of tourniquet remains popular during TKA for the benefits of decreasing immediate intraoperative blood loss and providing a clearer surgical field. However, after deflation of tourniquet, the dual effect of previous ischemia and surgically induced inflammation results in a reflex increase in blood flow and subsequent bleeding. The limb hypothermia caused by tourniquet use may also result in increased bleeding due to dysfunction of coagulatory enzymes. With these adverse effects on hemostasis, the net clinical effect of tourniquet use on perioperative hemoglobin remains undetermined.

Most of the current studies available directly compare only two tourniquet protocols on perioperative blood loss and/or change in perioperative hemoglobin level. The objective of this meta-analysis is to compile and compare the effects of all the currently used tourniquet protocols on perioperative change in hemoglobin level during primary TKA to determine if one protocol is superior.

\section{Materials and Methods}

\section{Techniques of tourniquet usage}

For the purpose of this study we grouped the currently utilized techniques of tourniquet usage during total knee arthroplasty in to 4 types (Table 1). No application or application and but no inflation of tourniquet throughout the procedure was considered type 0 . Type 1 technique is inflation of the tourniquet only during fixation of implants using cement and deflation immediately after setting of the cement. Inflation of the tourniquet just before the start of surgery and deflation to achieve hemostasis after fixation of implants and prior to 
Table 1: Techniques of tourniquet usage during total joint arthroplasty.

Type 0: No application or application but no inflation of tourniquet throughout the procedure.

Type 1: Inflation of the tourniquet only during fixation of implants using cement and deflation after setting of the cement.

Type 2: Inflation of the tourniquet just before the start of surgery and deflation to achieve hemostasis after fixation of implants and prior to closure of the arthrotomy.

- Type 2a - Wound closure without reinflation of tourniquet.

- Type $2 b$ - Wound closure by reinflation of tourniquet until completion of the procedure and application of the final dressing.

Type 3: Inflation of the tourniquet just before the start of surgery and deflation after closure of arthrotomy or complete closure of the surgical wound.

closure of the arthrotomy was considered type 2. Type 2 technique is again subdivided in to two based on whether surgical wound closure was performed without reinflation of tourniquet (type 2a) or with reinflation of tourniquet until completion of the procedure and application of the final dressing (type $2 \mathrm{~b}$ ). Finally, type 3 technique was defined as inflation of the tourniquet just before the start of surgery and deflation after closure of arthrotomy or complete closure of the surgical wound.

\section{Inclusion criteria}

This meta-analysis was conducted to investigate if there is any difference in the effect of various techniques of tourniquet usage on perioperative blood loss, specifically the change in the perioperative hemoglobin levels in patients undergoing primary knee replacement. Unpublished data of TKA done from May 2000 to March 2002 by one of the senior authors (FDC) using tourniquet application technique type $2 \mathrm{~b}$ ( 85 patients) and type 3 (63 patients) was included in the study and was considered as one record. Only patients undergoing unilateral primary total knee arthroplasty for osteoarthritis were included in either of the study groups.

Pub Med search was performed with the term 'Tourniquet Blood Loss Knee Replacement' without applying any additional filters to identify the records. In the same way, Embase search was performed subsequently using the same search terms and identified all the records. The duplicates among Pub Med and Embase search were identified and removed. The selected records were screened by reviewing the abstract for data pertaining specifically to the effect of tourniquet on perioperative blood loss in primary TKA and included for further analysis. If the abstract was not available for a record, it was selected for full-text review by default. Of all the records selected for full-text review, the original articles which had specific data on the effect of the tourniquet on preoperative and postoperative hemoglobin level or a change in the perioperative hemoglobin level in patients undergoing primary TKA were included for final analysis. In addition we looked carefully to identify additional records which we may have missed during Pub Med or Embase search but have the data that we are interested in by reviewing the references section of all the full-text reviewed records and if such a record was found, it was included in the final analysis.

\section{Exclusion criteria}

Any study that had patients who received an intra-operative blood transfusion was excluded, as it would affect the measured postoperative hemoglobin level. Also, studies that documented the use of tranexamic acid perioperatively for decreasing blood loss were excluded in this study. In addition the studies that were done exclusively in patients who underwent bilateral TKA were excluded. Finally, studies in which the groups of patients analyzed were not identical, for example, because of use of bipolar blood sealing systems, local infiltration or spraying of any pharmacological agent to control bleeding, and use of drain or cell saver in one but not the other studied group(s) were also excluded from further analysis.

\section{Statistics}

Initially we calculated the mean change in the perioperative hemoglobin while using a specific technique of tourniquet in each of the selected articles by subtracting mean postoperative hemoglobin recorded (before giving any blood transfusion) from the mean preoperative hemoglobin. Next step was to calculate the weighted mean of change in the perioperative hemoglobin level after combining the results of each specific technique of tourniquet usage from all the selected studies using the formula,

$$
\bar{x}_{w}=\frac{\sum_{i=1}^{n} w_{i} \bar{x}_{i}}{\sum_{i=1}^{n} w_{i}}
$$

where $\bar{x}_{w}$ is the weighted mean, $w_{1}$ is the weight for the $i^{\text {th }}$ observation, $\bar{x}_{i}$ is the mean of $i^{\text {th }}$ observation. Third part of the analysis was to calculate the weighted variance of change in the mean perioperative hemoglobin level after combining the results of each specific technique of tourniquet usage from all the selected studies using the formula,

$$
s_{w}^{2}=\frac{\sum_{i=1}^{n} w_{i}\left(x_{i}-\bar{x}_{w}\right)^{2}}{\frac{\left(N^{\prime}-1\right) \sum_{i=1}^{n} w_{i}}{N^{\prime}}}
$$

where $s_{w}^{2}$ is the weighted variance, $w_{1}$ is the weight for the $i^{\text {th }}$ observation, $x_{1}$ is the mean of $i^{\text {th }}$ observation, $\bar{x}_{w}$ is the weighted mean and $N^{\prime}$ is the number of weights. The fourth step was to calculate the weighted standard deviation of change in the mean perioperative hemoglobin level after combining the results of each specific technique of tourniquet usage from all the selected studies using the formula,

$$
s d_{w}=\sqrt{\frac{\sum_{i=1}^{n} w_{i}\left(x_{i}-\bar{x}_{w}\right)^{2}}{\frac{\left(N^{\prime}-1\right) \sum_{i=1}^{n} w_{i}}{N^{\prime}}}}
$$

where $s d_{w}$ is the weighted standard deviation, $w_{1}$ is the weight for the $i^{\text {th }}$ observation, $x_{1}$ is the mean of $i^{\text {th }}$ observation, $\bar{x}_{w}$ is the weighted mean and $N^{\prime}$ is the number of weights. Weighted standard error of change in the mean perioperative hemoglobin level after combining the results of each specific technique of tourniquet usage from all the selected studies using the formula,

$$
S E_{w}=\frac{s d_{w}}{\sqrt{N^{\prime}}}
$$

where $S E_{w}$ is the weighted standard error, $s d_{w}$ is the weighted standard deviation and $N^{\prime}$ is the number of weights. The final step was to calculate the $95 \%$ confidence interval (CI) of the weighted meanchange in the mean perioperative hemoglobin level after combining the results of each specific technique of tourniquet usage from all the selected studies using the formula, $\bar{X}_{w} \pm 1.96 \times S E_{w}$, where $\bar{X}_{w}$ is the weighted mean and $S E_{w}$ is the weighted standard error. 


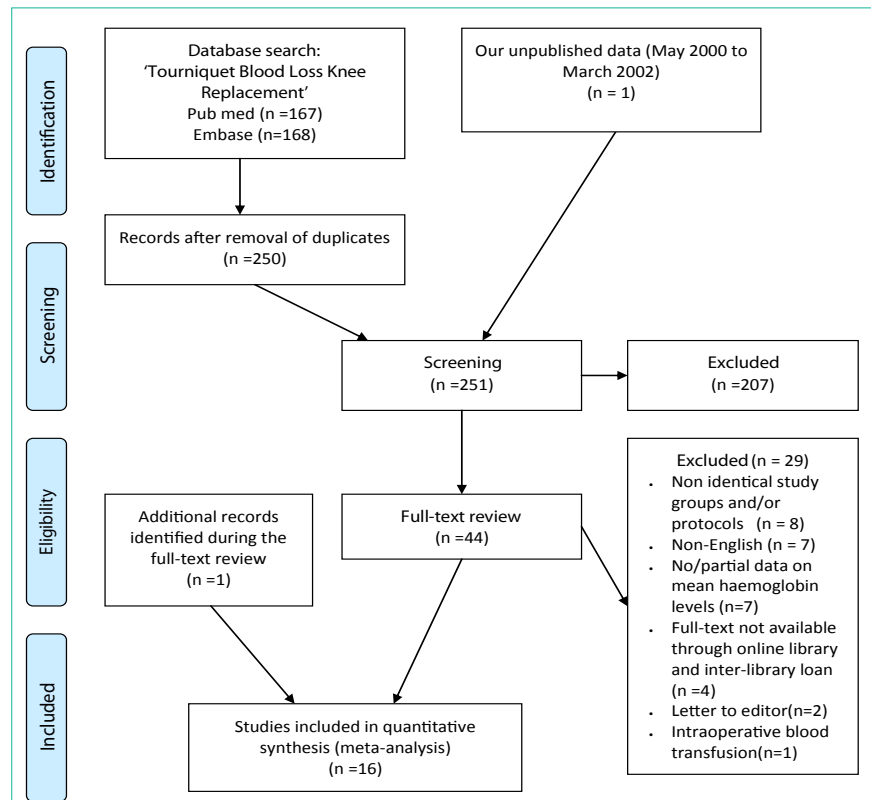

Figure 1: The PRISMA Diagram showing the study flow from identification of original articles through meta-analysis.

\section{Results}

We identified 167 records in Pub Med and 168 records in Embase using the search term 'Tourniquet Blood Loss Knee Replacement' (Figure 1). After removing the duplicates, there were 250 articles available for screening of abstracts. When we included our unpublished study as one additional record, we had a total of 251 records available for screening. We excluded 207 records among the 251 that were screened as they were not relevant to this study. We full-text reviewed the remaining 44 records among the 251 records that were screened. Out of 44 records that were full text reviewed, 29 records were excluded for various reasons. Hence we identified a total of 15 original articles with data relevant to this study. During the process of full text review of these 44 records, we could identify one additional original article that was not previously identified by either Pub Med or Embase search, but had the data comparing the effect of two different techniques of using tourniquet on change in the mean perioperative a hemoglobin level during primary TKA. Including this one newly identified original article, we had a total of 16 articles available for meta-analysis.

The total number of patients available for analysis was 136 for type 0 ; 97 for type 1; 398 for type 2a; 134 for type $2 \mathrm{~b}$; and 438 for type 3 tourniquet usage (Table 2). The calculated mean change in the hemoglobin perioperatively in each of the study groups in each individual study is shown in Table 2. The weighted mean, weighted variance, weighted standard deviation, weighted standard error and 95\% CI of the weighted mean change in the perioperative hemoglobin level (all in $\mathrm{g} / \mathrm{dl}$ ) after combining the results of each specific technique of tourniquet usage are represented in Table 3 . The results of type 2a, type $2 b$ and type 3 techniques of tourniquet usage during primary TKA were essentially comparable. Type $2 \mathrm{a}$, type $2 \mathrm{~b}$ and type 3 techniques of tourniquet usage were found to have the lowest mean change in the perioperative hemoglobin level and a non overlapping
95\% confidence interval of the mean change in the perioperative hemoglobin level compared to type 0 technique of tourniquet usage. Type 1 technique of tourniquet usage had the broadest $95 \%$ confidence interval indicating significant individual outliers compared to the rest of the techniques of tourniquet usage during primary TKA.

\section{Discussion}

This is the first study to classify various types of tourniquet usage during primary total knee arthroplasty. Also this is the first meta-analysis to compare the effect of all the different techniques of tourniquet usage on perioperative hemoglobin level in patients undergoing primary TKA, facilitating derivation of strongest possible evidence required to provide a clinical guideline.

Clinician should be able to differentiate between "statistical significance" and "clinical meaningfulness". In this study for example, the mean change in the perioperative hemoglobin level between type 0 technique $(3.67 \mathrm{~g} / \mathrm{dl})$ and type $3(2.90 \mathrm{~g} / \mathrm{dl})$ technique of tourniquet usage is the highest $(0.77 \mathrm{~g} / \mathrm{dl})$ when any two groups are compared with each other. Apparently it may not seem a clinically meaningful difference $(<1 \mathrm{~g} / \mathrm{dl})$ unless one critically evaluates two related issues. One, the hemoglobin loss while using type 0 technique is permanent compared to type 3 as the essential components for production of new red blood cells are lost forever, unless a cell saver device is used for reinfusion, which none the less comes only at an additional cost. Two, while performing a primary bilateral TKA, this difference in the effect size becomes double, and then may become clinically meaningful difference.

The type 3 technique of tourniquet usage during total knee arthroplasty needs to be carefully compared with the rest of the types of tourniquet usage in terms of the risk of unidentified major vessel injury. In three individual studies by Barwell $J$ [2], Hernández-Castaños [6] and Chirstodouou [16] calculated the odds of unrecognized major vessel injury in type 2a technique compared to type 3 and found it to be 0.08 ( $95 \%$ CI: 0.00 to 1.51), 0.23 ( $95 \%$ CI: 0.02 to 2.16 ) and 0.48 ( $95 \%$ CI: 0.04 to 5.66 ) respectively. As the $95 \%$ CI falls on either side of 1.00 in the results of all these three studies, it is not possible to conclude that releasing the tourniquet before wound closure is associated with less risk of unrecognized major vessel injury. However, clinical judgment matters in this situation. It is not difficult to identify a major bleeding vessel even while working with the tourniquet inflated, and if necessary, the surgeon should have little hesitation to deflate the tourniquet and achieve control over bleeding and proceed further with or without reinflation of tourniquet.

\section{Limitations}

The results of this study like any other meta-analysis have to be understood considering the heterogeneity of the patients groups that are compared. Though many filters have been used in this study to see that the patients groups that were compared are identical, it is impossible to eliminate all the confounding variables during a meta-analysis. For example, the type of anaesthesia (hypotensive epidural anaesthesia vs. general anaesthesia) can have a profound effect on perioperative blood loss, which we did not try to eliminate. In addition, there was no standardized deep venous thrombosis prophylaxis used for the patients among all the included studies. 
Table 2: The articles selected for meta-analysis (in alphabetical order of first author of study), number of patients included in each study group and mean change in the perioperative hemoglobin.

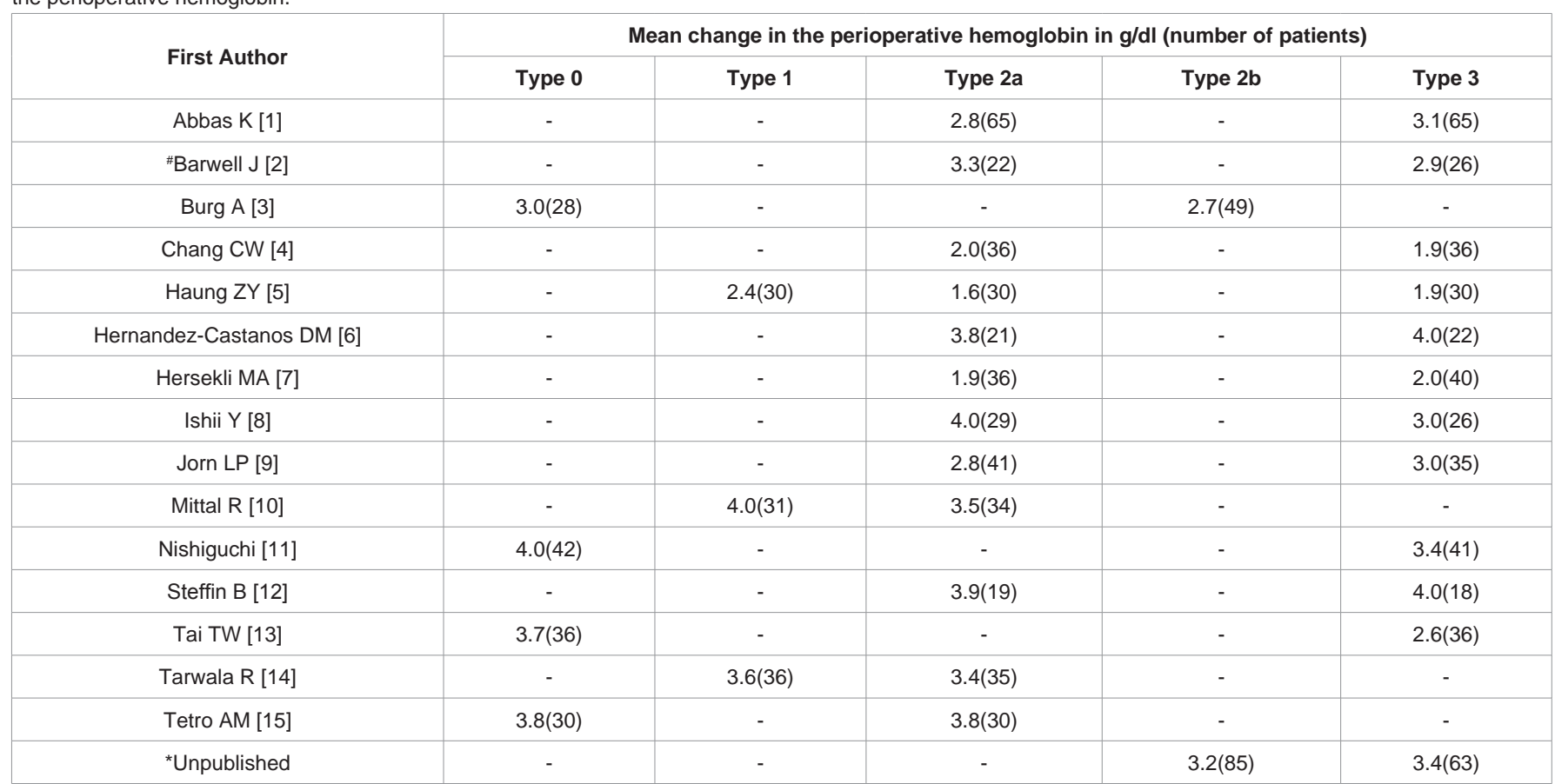

\#Values were expressed in median. Cases in which blood transfusion was given during surgery could be excluded from total number of patients as the data was available in that form. We considered median value to be equal to mean during analysis of results.

*Our unpublished data that is included now for the meta-analysis.

Table 3: The weighted mean, weighted variance and weighted standard deviation, weighted error and $95 \% \mathrm{Cl}$ of the mean change in the perioperative hemoglobin (in $\mathrm{g} / \mathrm{dl})$ of each type of tourniquet usage.

\begin{tabular}{|c|c|c|c|c|c|}
\hline Technique of tourniquet usage & Weighted mean & Weighted variance & Weighted standard deviation & Weighted error & 95\% CI (Lower to Upper limit) \\
\hline Type 0 & 3.67 & 0.21 & 0.46 & 0.03 \\
\hline Type 1 & 3.35 & 0.65 & 0.80 & 0.46 \\
\hline Type 2a & 2.96 & 0.64 & 0.80 & 0.29 \\
\hline Type 2b* & 3.01 & 0.11 & 0.34 & 2.44 to 4.25 \\
\hline Type 3 & 2.90 & 0.44 & 0.66 & 2.95 to 3.07 3.53 & 0.03 \\
\hline
\end{tabular}

\section{Conclusions}

Type $2 \mathrm{a}$, type $2 \mathrm{~b}$ and type 3 techniques of tourniquet usage have comparable effect on change in the perioperative hemoglobin levels in patients undergoing primary total knee arthroplasty. Type 0 technique of tourniquet usage is inferior to type2a, type $2 \mathrm{~b}$ and type 3 techniques of tourniquet usage in terms of loss of the hemoglobin perioperatively. Type 1 technique has significant individual outliers compared to rest of the techniques of tourniquet usage during primary TKA indicating less reliability on a case-to-case basis compared to rest of the techniques. Hence to conserve the blood loss during primary total knee arthroplasty, we recommend either type 2a or type $2 \mathrm{~b}$ or type 3 as the most preferable technique of usage.

\section{Scope for Future Research}

Prevention of blood loss during total knee arthroplasty is a multipronged strategy. Introduction of tranexamic acid in arthroplasty surgery to prevent blood loss has been proven to be advantageous. There are several other potentially effective locally applied strategies for prevention of blood loss during primary TKA. Hence future studies should focus on comparing superiority and cost effectiveness of one specific protocol with another to develop the most economic yet the most efficient strategy for complete elimination of need for perioperative blood transfusion in patients undergoing primary TKA.

\section{References}

1. Abbas K, Raza H, Umer M, Hafeez K. Effect of early release of tourniquet in total knee arthroplasty. J Coll Physicians Surg Pak. 2013; 23: 562-565.

2. Barwell J, Anderson G, Hassan A, Rowlings I. The effects of early tourniquet release during total knee arthroplasty: a prospective randomised double-blind study. J Bone Joint Surg Br. 1997; 79: 265-268.

3. Burg A, Dudkiewicz I, Heller S, Salai M, Velkes S. The effects of using a tourniquet in total knee arthroplasty: A study of 77 patients. Journal of Musculoskeletal Research. 2009; 12: 137.

4. Chang CW, Lan SM, Tai TW, Lai KA, Yang CY. An effective method to reduce ischemia time during total knee arthroplasty. J Formos Med Assoc. 2012; 111: 19-23.

5. Huang ZY, Pei FX, Ma J, Yang J, Zhou ZK, Kang PD, et al. Comparison of three different tourniquet application strategies for minimally invasive total knee arthroplasty: a prospective non-randomized clinical trial. Arch Orthop Trauma Surg. 2014; 134: 561-570.

6. Hernández-CastañosDM, Ponce VV, Gil F. Release of ischaemia prior to 
wound closure in total knee arthroplasty: a better method? IntOrthop. 2008; 32: $635-638$

7. Hersekli MA, Akpinar S, Ozkoc G, Ozalay M, Uysal M, Cesur N, et al. The timing of tourniquet release and its influence on blood loss after total knee arthroplasty. IntOrthop. 2004; 28: 138-141.

8. Ishii $Y$, Matsuda $Y$. Effect of the timing of tourniquet release on perioperative blood loss associated with cement less total knee arthroplasty: a prospective randomized study. J Arthroplasty. 2005; 20: 977-983

9. Jorn LP, Lindstrand A, Toksvig-Larsen S. Tourniquet release for hemostasis increases bleeding. A randomized study of 77 knee replacements. 1999; 70 : 265-267.

10. Mittal R, Ko V, Adie S, Naylor J, Dave J, Dave C, et al. Tourniquet application only during cement fixation in total knee arthroplasty: a double-blind, randomized controlled trial. ANZ J Surg. 2012; 82: 428-433.

11. Nishiguchi M, Takamura N, Kono M, Aoyagi K. Estimation of blood loss in tota knee arthroplasty with and without tourniquet. ActamedicaNagasakiensia. 2008; 53: 105-109.
12. Steffin B, Green-Riviere E, Giori NJ. Timing of tourniquet release in total knee arthroplasty when using a postoperative blood salvage drain.J Arthroplasty. 2009; 24: 539-542.

13. Tai TW, Chang CW, Lai KA, Lin CJ, Yang CY. Effects of tourniquet use on blood loss and soft-tissue damage in total knee arthroplasty: a randomized controlled trial. J Bone Joint Surg Am. 2012 19; 94: 2209-2215.

14. Tarwala R, Dorr LD, Gilbert PK, Wan Z, Long WT. Tourniquet use during cementation only during total knee arthroplasty: a randomized trial. ClinOrthopRelat Res. 2014; 472: 169-174.

15. Tetro AM, Rudan JF. The effects of a pneumatic tourniquet on blood loss in total knee arthroplasty. Can J Surg. 2001; 44: 33-38.

16. Christodoulou AG, Ploumis AL, Terzidis IP, Chantzidis P, Metsovitis SR, Nikiforos DG. The role of timing of tourniquet release and cementing on perioperative blood loss in total knee replacement. Knee. 2004; 11: 313-317.
Austin J Musculoskelet Disord - Volume 4 Issue 1 - 2017

ISSN : 2381-8948 | www.austinpublishinggroup.com

Boddu et al. (C) All rights are reserved
Citation: Boddu C, Nett MP, Cushner FD and Scuderi GR. Blood Loss in Total Knee Arthroplasty: A Metaanalysis of 4 Different Tourniquet Protocols. Austin J Musculoskelet Disord. 2017; 4(1): 1041. 